\title{
Dispersive Charge and Flux Qubit Readout as a Quantum Measurement Process
}

\author{
Lars Tornberg and Göran Johansson \\ Applied Quantum Physics, MC2, Chalmers, S-412 96 Göteborg, Sweden
}

\begin{abstract}
We analyze the dispersive readout of superconducting charge and flux qubits as a quantum measurement process. The measurement oscillator frequency is considered much lower than the qubit frequency. This regime is interesting because large detuning allows for strong coupling between the measurement oscillator and the signal transmission line, thus allowing for fast readout. Due to the large detuning we may not use the rotating wave approximation in the oscillator-qubit coupling. Instead we start from an approximation where the qubit follows the oscillator adiabatically, and show that non-adiabatic corrections are small. We find analytic expressions for the measurement time, as well as for the back-action, both while measuring and in the offstate. The quantum efficiency is found to be unity within our approximation, both for charge and flux qubit readout.
\end{abstract}

PACS numbers: 74.50.+r 03.65.-w 03.67.Lx 42.50.Lc

\section{INTRODUCTION}

Superconducting qubits are considered one of the technologies capable of bringing us quantum information processing (QIP) devices in the future. ${ }^{1}$ One cornerstone of QIP is the possibility to convert the quantum state into classical information, i.e. read-out. A large effort has gone into optimizing different read-out techniques, see e.g. Ref. 2 and references therein. Of course reading out a qubit only makes sense if it can stay coherent long enough to contain true quantum information. Being solid-state qubits, superconducting qubits interact rather strongly with their environment, making dephasing the worst enemy of superconducting QIP. 


\section{Tornberg and G. Johansson}

Quite naturally, which type of noise is most dangerous depends on the qubit design. For example charge qubits ${ }^{3}$ are most sensitive to charge fluctuations, ${ }^{4}$ while flux qubits are most sensitive to flux noise ${ }^{5}$ and charge-phase qubits $^{6}$ are sensitive to both. ${ }^{7}$ At the degeneracy point the two charge/flux qubit states have equal average charge/flux. Thus, at this point they are protected against environmental charge/flux noise to first order in the coupling. To minimize the effect of the typical noise, qubits are now operated so that they remain at this degeneracy point as much as possible.

The natural readout for a charge/flux qubit is to measure the average charge/flux of the qubit. Since the qubit states have equal average charge/flux at the degeneracy point, the natural type of readout is no longer possible. This initiated an interest in dispersive readout techniques, where the qubit state influences the properties of a harmonic oscillator, which is then probed..$^{8-10}$

In the experiment of Wallraff et. al. at Yale, ${ }^{10}$ a charge qubit was coupled to an oscillator, realized by a coplanar waveguide. Using this setup they managed to determine the state of the qubit, while maintaining long coherence times. In this case, the oscillator and qubit where nearly degenerate in frequency and an oscillator with a high quality factor $Q$ was used to shield the qubit from relaxation.

In this paper, we concentrate on the situation where the qubit and oscillator frequencies differ by orders of magnitude. This will allow us to use a low $Q$ oscillator, thereby increasing the read-out speed of the circuit. Here it is the detuning that shields the qubit from measurement induced mixing, and also allows relaxation to be minimized through effective filtering of the transmission line at the qubit frequency. In this setup the state of a charge/phase qubit can be read out through its effective capacitance/inductance, which shifts the resonant frequency. At the degeneracy point, the state dependent quantum capacitance/inductance for the two states have the same magnitude but differ in sign. For the charge qubit the quantum capacitance was recently measured by two groups. ${ }^{11,12}$

The theory of quantum measurement states the impossibility to discern the state of a quantum system without destroying the phase coherence in the system. This promotes the idea of defining the quantum efficiency $\eta$ of a measurement as the ratio between the dephasing time $t_{\varphi}$ and measurement time $t_{m s}$, bounded above by $\eta=t_{\varphi} / 2 t_{m s} \leq 1 .{ }^{13}$ (To be consistent with the results of the Yale group, ${ }^{16}$ we have decided to use their definition of measurement time. This, together with the desire to keep $\eta \leq 1$ is the reason for the appearance of the one half in the definition). The quantum efficiency gives a measure on the back-action of the read-out and deriving $\eta$ in terms of circuit parameters is thus a good starting point for optimizing the read-out. 


\section{Quantum efficiency of charge and flux qubit readout}

In Ref. 14, together with C. M. Wilson, we investigated the quantum efficiency of the read-out proposal mentioned above, as well as discussed the performance of a realistic circuit design. Here we give the more detailed theoretical background for those results. The large detuning between the qubit and oscillator makes standard methods of quantum optics ${ }^{15,16}$ inappropriate. Instead, we start from an approximation where the qubit follows the oscillator adiabatically, and show that non-adiabatic corrections are small. Our results are based on the quantum network theory, introduced by Yurke and Denker. ${ }^{17}$ As already discussed in Ref. 14, the efficiency for both charge and phase qubit readout is ideal within our approximation. This result is independent of the oscillator $Q$-value. Furthermore, we find that a low $Q$ oscillator shields the qubit from thermal dephasing. Finally we treat explicitly the limiting case of a transmission line capacitively coupled directly to a charge qubit. Being of more academic interest, we find an ideal quantum efficiency also in this case.

\section{CHARGE QUBIT}

The goal of this section is to derive the Hamiltonian for the superconducting charge qubit complete with read-out device. Having the full quantum description of the circuit we then make suitable approximations to derive the quantum capacitance of the Cooper-pair box. This gives us an adiabatic Hamiltonian which we can solve to obtain the dynamics of the system exactly. In section 4. we give bounds for the non-adiabatic contributions, and justify our approximations in detail. In the rest of the paper we use the adiabatic Hamiltonian to investigate the efficiency of our measurement scheme.

The single Cooper-pair box operated as a charge qubit ${ }^{18}$ can be viewed in

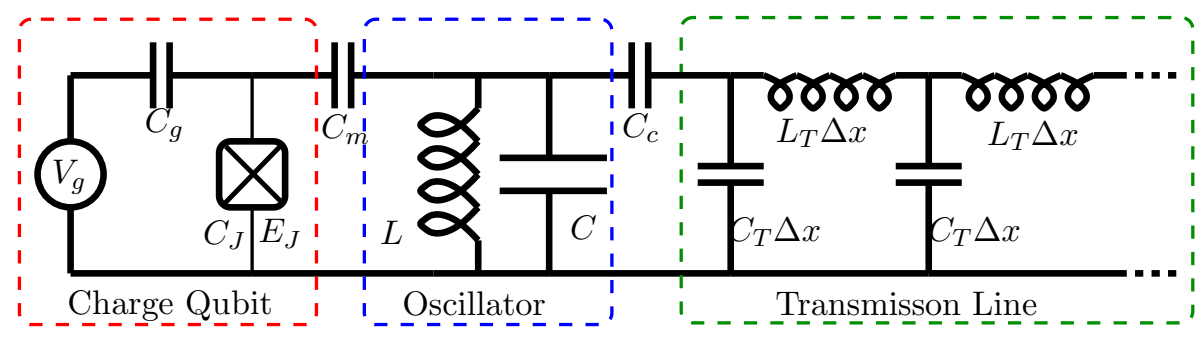

Fig. 1. Cooper-pair box operated as a charge-qubit complete with read-out circuitry, see text. 


\section{Tornberg and G. Johansson}

Fig. 1] A small superonducting island is connected to a superconducting lead through a Josephson junction with Josephson energy $E_{J}$ and capacitance $C_{J}$. The island is also coupled to a gate voltage $V_{g}$ via the gate capacitance $C_{g}$. By tuning $V_{g}$, the desired working point of the qubit can be reached. To enable readout an LC-oscillator is coupled to the box via a measuring capacitance $C_{m}$. The oscillator is in turn coupled to a transmission line, here modelled as a semi-infinite line of LC-oscillators in parallel. The line is characterized by its capacitance $C_{T}$ and inductance $L_{T}$ per unit length. Through this line, all measurements of the qubit will be performed.

We choose, as our starting point, to write down the Lagrangian for the system. ${ }^{17,19}$ This method gives automatically the conjugated variables of the system and thus provides us with the dynamics. Since the circuit contains a Josephson element the natural choice of coordinates is the phase across each circuit element, which is related to the voltage drop by $\phi_{i}=\int V_{i} d t$. Following standard procedure ${ }^{19}$ the capacitive and inductive energy of the circuit are the kinetic and potential energy of the Lagrangian

$$
\begin{aligned}
T & =\frac{C_{g} \dot{\Phi}_{g}^{2}}{2}+\frac{C_{J} \dot{\Phi}_{J}^{2}}{2}+\frac{C_{m} \dot{\Phi}_{m}^{2}}{2}+\frac{C \dot{\Phi}_{C}^{2}}{2}+\frac{C_{c} \dot{\Phi}_{i n}^{2}}{2}+\sum_{i=1}^{\infty} \Delta x \frac{C_{T}\left(\dot{\Phi}_{i}^{p}\right)^{2}}{2} \\
V & =\frac{\Phi_{L}^{2}}{2 L}-E_{J} \cos \left(\frac{\Phi_{J}}{\Phi_{0}}\right)+\sum_{i} \Delta x \frac{\left(\Phi_{i+1}^{p}-\Phi_{i}^{p}\right)^{2}}{2 L_{T}(\Delta x)^{2}}
\end{aligned}
$$

where $\Phi_{0}=\hbar / 2 e$ is the flux quantum. Applying Kirchoff's voltage law gives the constraints on the circuit coordinates which gives the Lagrangian, $L=T-V$, for the system

$$
\begin{aligned}
L & =\frac{C_{q b} \dot{\Phi}_{J}^{2}}{2}+\frac{\left(C_{o s c}+C_{c}\right) \dot{\Phi}_{C}^{2}}{2}+\frac{C_{c}\left(\dot{\Phi}_{1}^{p}\right)^{2}}{2}-\frac{\Phi_{C}^{2}}{2 L}+E_{J} \cos \left(\frac{\Phi_{J}}{\Phi_{0}}\right)- \\
& -C_{m} \dot{\Phi}_{C} \dot{\Phi}_{J}-C_{c} \dot{\Phi}_{C} \dot{\Phi}_{1}^{p}-C_{g} V_{g} \dot{\Phi}_{J}+\frac{C_{g} V_{g}^{2}}{2}+ \\
& +\sum_{i=1}^{\infty} \Delta x\left(\frac{C_{T}\left(\dot{\Phi}_{i}^{p}\right)^{2}}{2}-\frac{\left(\Phi_{i+1}^{p}-\Phi_{i}^{p}\right)^{2}}{2 L_{T}(\Delta x)^{2}}\right),
\end{aligned}
$$

where we have introduced the capacitances $C_{q b}=C_{J}+C_{g}+C_{m}$, and $C_{\text {osc }}=C+C_{m}$ for brevity. To simplify the derivation of the Hamiltonian we introduce the vector notation for our coordinates

$$
\vec{\Phi}=\left[\begin{array}{lllll}
\Phi_{J} & \Phi_{C} & \Phi_{1}^{p} & \Phi_{2}^{p} & \cdots
\end{array}\right] .
$$

The Lagrangian can now be written more compactly

$$
L=\frac{1}{2} \dot{\vec{\Phi}}^{T} C \dot{\vec{\Phi}}-C_{g} V_{g} \dot{\Phi}_{J}-\frac{\Phi_{C}^{2}}{2 L}+E_{J} \cos \left(\frac{\Phi_{J}}{\Phi_{0}}\right)-\sum_{i} \Delta x \frac{\left(\Phi_{i+1}^{p}-\Phi_{i}^{p}\right)^{2}}{2 L_{T}(\Delta x)^{2}}
$$




\section{Quantum efficiency of charge and flux qubit readout}

where $C$ is the mass matrix of the system. This can be divided into $C_{s y s}$, describing the capacitative elements in the qubit-oscillator circuit and a pure diagonal matrix $C_{T L}$ which only contains information about the capacitance per unit length in the transmission line

$$
\begin{aligned}
C & =\left[\begin{array}{c|c}
C_{\text {sys }} & 0 \\
\hline 0 & C_{T L}
\end{array}\right], \quad C_{\text {sys }}=\left[\begin{array}{ccc}
C_{q b} & -C_{m} & 0 \\
-C_{m} & C_{o s c}+C_{c} & -C_{c} \\
0 & -C_{c} & C_{c}+C_{T} \Delta x
\end{array}\right], \\
C_{T L} & =\left[\begin{array}{ccc}
C_{T} & 0 & \cdots \\
0 & C_{T} & \ddots \\
\vdots & \ddots & \ddots
\end{array}\right] .
\end{aligned}
$$

The Lagrangian contains all information needed for deriving the system dynamics. However, when doing quantum mechanical calculations one often prefers to work with the Hamiltonian. We thus Legendre transform $^{20}$ the Lagrangian following Appendix A. and arrive at

$$
H=H_{q b}+H_{o s c}+H_{i n t}+H_{T L},
$$

where $H_{q b}$ describes the Josephson junction alone and also the interaction between JJ and oscillator

$$
H_{q b}=\frac{D_{11}}{2}\left(p_{J}+C_{g} V_{g}\right)^{2}+\left(D_{12} p_{C}+D_{13} p_{p}\right)\left(p_{J}+C_{g} V_{g}\right)-E_{J} \cos \left(\frac{\Phi_{J}}{\Phi_{0}}\right),
$$

and where $D_{i j}$ are the elements of the matrix

$$
D=C_{s y s}^{-1}=\frac{1}{C_{q b} C_{o s c}-C_{m}^{2}}\left[\begin{array}{ccc}
C_{o s c} & C_{m} & C_{m} \\
C_{m} & C_{q b} & C_{q b} \\
C_{m} & C_{q b} & \left(C_{q b} C_{o s c}-C_{m}^{2}\right) / C_{c}+C_{q b}
\end{array}\right]
$$

The last three terms in the Hamiltonian, Eq. (6), describe the oscillator, the transmission line and the coupling between them;

$$
\begin{aligned}
& H_{\text {osc }}=\frac{D_{22}}{2} p_{C}^{2}+\frac{\Phi_{C}^{2}}{2 L}, \quad H_{\text {int }}=D_{23} p_{C} p_{p}, \\
& H_{T L}=\frac{D_{33}}{2} p_{p}^{2}+\frac{1}{\Delta x} \sum_{i=1}^{\infty}\left(\frac{\left(p_{(i+1)}^{p}\right)^{2}}{2 C_{T}}+\frac{\left(\Phi_{i+1}^{p}-\Phi_{i}^{p}\right)^{2}}{2 L_{T}}\right),
\end{aligned}
$$

where the operators $p_{C}, p_{J}, p_{p}$ and $p_{i}^{p}$ are the conjugate momenta to $\Phi_{C}, \Phi_{J}, \Phi_{1}^{p}$ and $\Phi_{i}^{p}$ respectively, and have dimension of charge. By definition, the phases and charges $\phi_{i}$ and $p_{j}$ obey the canonical commutations relation $\left[\phi_{i}, p_{j}\right]=i \hbar \delta_{i j}$. The kinetic energy of the Hamiltonian is thus represented 


\section{Tornberg and G. Johansson}

by the the charging energies of the circuit. The $D$-matrix contains information about the effective capacitances associated with the respective charges.

The JJ island is caracterized by a charging energy, $E_{C}=D_{11} e^{2} / 2 \approx$ $e^{2} / 2 C_{q b}$ and the Josephson energy $E_{J}$. To realize a two level system, the charging energy of the island needs to be much larger than the Josephson energy. For this choice of parameters we can limit the number of excess electrons on the island to the values $\{0,2\}$. Projecting the qubit Hamiltonian on this subspace of the whole Hilbert space in charge basis, we get the usual qubit Hamiltonian in the language of Pauli spin matrices

$$
H_{q b}=-\frac{E_{e l}}{2} \sigma_{z}-\frac{E_{J}}{2} \sigma_{x}+2 E_{c} \kappa\left(n_{C}+n_{p}\right)\left(1-n_{0}\right)+E_{C}\left(2-n_{0}^{2}\right),
$$

where $\kappa=D_{12} / D_{11}=C_{m} / C_{\text {osc }}$ is a dimensionless coupling constant and $E_{e l}=4 E_{c}(1-\tilde{n})$ is the electrostatic energy of the island depending on both gate charge and induced charge from the oscillator and transmission line $\tilde{n}=n_{0}+\kappa\left(n_{C}+n_{p}\right)$. The number operators $n$ are related to the charges by $n_{C}=p_{C} / e, n_{p}=p_{p} / e$ and $n_{0}=C_{g} V_{g} / e$ respectively. The last term in Eq. (10) is just an offset in the energy of the system and thus discarded.

\subsection{QUANTUM CAPACITANCE}

During read-out the oscillator will be driven close to the bare resonance frequency which we consider much smaller than the qubit level-splitting $E_{J} / \hbar$. This difference in frequency allows us to make an adiabatic approximation, separating the qubit and oscillator dynamics. Thus the qubit eigenvalues and eigenstates will depend on the oscillator and transmission line coordinates. However the levels will never cross and the qubit is frozen in its state. In this regime, the qubit can be described by an effective adiabatic Hamiltonian

$$
H_{\mathrm{A}}=\frac{\sqrt{E_{e l}(\tilde{n})^{2}+E_{J}^{2}}}{2} \sigma_{z}+2 E_{c} \kappa\left(n_{C}+n_{p}\right)\left(1-n_{0}\right),
$$

which is obtained by a unitary transformation of the the qubit Hamiltonian in Eq. (10). For more details on this, see Sec. 4. The expression in Eq. (11) is similar to the usual expression for the Hamiltonian of a charge qubit in computational basis. The difference in this case is that the working point of the qubit is determined not only by the $V_{g}$ dc-bias, but also on the induced charge on the oscillator. The second term is usually proportional to the identity operator and ignored. Here it contains both system and transmission line operators, and can not be thrown away. However, it only represents a 


\section{Quantum efficiency of charge and flux qubit readout}

small shift in $p_{C}$ and $p_{1}$ and can thus be included into a new set of shifted operators. Considering a small coupling between qubit and oscillator we can expand the qubit energy to second order in $\delta n=\kappa\left(n_{C}+n_{p}\right)$. The effect of the qubit can thus be summarized in three terms; one state dependent off-set which does not affect the circuit dynamics, a linear term in $\kappa\left(n_{C}+\right.$ $n_{p}$ ) which is zero at the degeneracy point, and finally a term quadratic in oscillator and transmission line charge. Discarding the two first we arrive at the Hamiltonian determining the low frequency dynamics of the oscillator and transmission line, at the charge degeneracy point

$$
\begin{aligned}
H & =\frac{D_{22}}{2} p_{C}^{2}+\frac{\Phi_{C}^{2}}{2 L}+\frac{g_{C}}{2} \sigma_{z}\left(p_{C}+p_{p}\right)^{2} \\
& +\frac{D_{33}}{2} p_{p}^{2}+\frac{1}{\Delta x} \sum_{i}\left(\frac{\left(p_{(i+1)}^{p}\right)^{2}}{2 C_{T}}+\frac{\left(\Phi_{i+1}^{p}-\Phi_{i}^{p}\right)^{2}}{2 L_{T}}\right)+D_{23} p_{C} p_{p} .
\end{aligned}
$$

Here we have defined the coupling between the charge qubit and oscillator $g_{C}=C_{Q} / C_{o s c}^{2}=\frac{8 E_{c}^{2} \kappa^{2}}{e^{2} E_{J}}$, and the quantum capacitance as

$$
C_{Q}=-\frac{2 e^{2} C_{m}^{2}}{E_{J} C_{q b}^{2}} \sigma_{z} .
$$

The motivation behind this definition comes from the fact that the qubit shifts the electrostatic energy of the oscillator, which can be interpreted as a shift of the oscillator capacitance, $C_{o s c} \rightarrow C_{o s c}+C_{Q}$. This interpretation is possible as long as $C_{Q} \ll C_{o s c}$. The corresponding qubit induced shift of the oscillators resonance frequency is simply given by $\omega_{\text {osc }}=1 / \sqrt{L\left(C_{o s c}+C_{Q}\right)}$.

\section{FLUX QUBIT AND QUANTUM INDUCTANCE}

The radio-frequency superconducting quantum interference device (rfSQUID) operated as a flux qubit ${ }^{21}$ can be seen in Fig. 2. The readout part of our analysis also applies to the 3 -junction persistent current qubit, ${ }^{5}$ but the rf-SQUID is chosen for simplicity. In the previous section the charge states of the single Cooper-pair box were used as the computational basis of the qubit. Here the qubit loop is biased by an external flux $\Phi_{x}$, and the $|0\rangle$ and $|1\rangle$ states are realized by the clock and anticlockwise circulating supercurrents in the loop. The read-out circuit is the same as for the charge qubit. It is however inductively coupled to the qubit via the mutual inductance $M$, as opposed to the capacitative coupling in the charge case. The derivation of the Hamiltonian for this circuit is almost analogous to the analysis for the charge qubit. Making the adiabatic approximation and rotating to the qubit 


\section{Tornberg and G. Johansson}

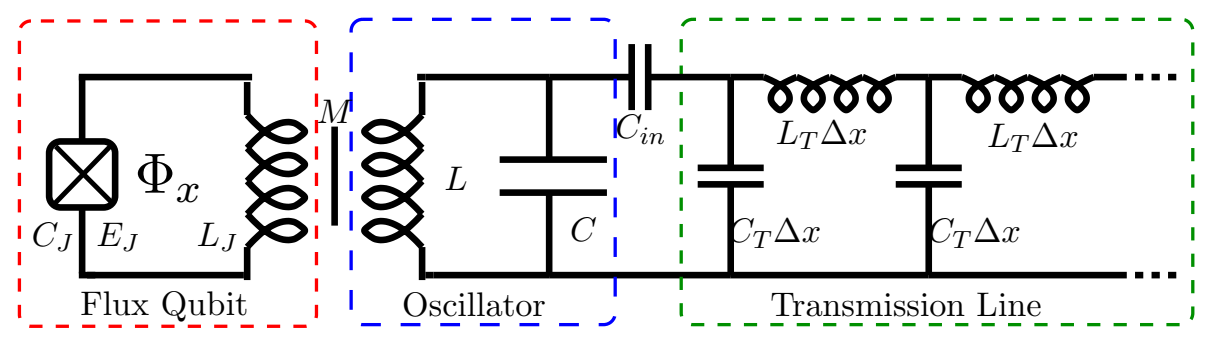

Fig. 2. Radio-frequency SQUID operated as a flux-qubit, complete with read-out circuitry, see text.

eigenbasis yields the following adiabatic Hamiltonian for the oscillator and transmission line, considering the qubit biased at its degeneracy point

$$
\begin{aligned}
H & =\frac{p^{2}}{2 C}+\left(\frac{1}{2 L}-\frac{M^{2}\langle\phi\rangle^{2}}{\Delta\left(L L_{J}\right)^{2}} \sigma_{z}\right) \Phi^{2}+\frac{\left(p_{p}\right)^{2}}{2 C_{c}}+\frac{p_{p} p}{C}+ \\
& +\frac{1}{\Delta x} \sum_{i}\left(\frac{\left(p_{(i+1)}^{p}\right)^{2}}{2 C_{T}}+\frac{\left(\Phi_{i+1}^{p}-\Phi_{i}^{p}\right)^{2}}{2 L_{T}}\right)
\end{aligned}
$$

where $C_{c}=\left(C+C_{i n}\right) /\left(C C_{i n}\right),\langle\phi\rangle$ is the magnitude of the flux generated by the qubit persistent current in a current eigenstate, and $\Delta$ is the qubit energy splitting. Just as in the charge case, the slow transverse coupling to the oscillator is seen as a longitudinal coupling in the eigenbasis of the qubit. Due to the phase-coupling between oscillator and qubit we get the coupling constant $g_{L}=L_{Q} / L^{2}$ where the quantum inductance is defined as

$$
L_{Q}=\frac{M^{2}\langle\phi\rangle^{2}}{\Delta L_{J}^{2}}
$$

so that the coupling can be incorporated into an effective inductance $L^{g / e} \equiv$ $L \pm L_{Q}$.

\section{NON-ADIABATIC CORRECTIONS AND QUBIT RELAXATION}

In the following we will calculate the dynamics of the oscillator and transmission line, considering the qubit state to be fixed in its adiabatic eigenbasis. This is of course an approximation, and there are two different effects induced by the measurement circuit that will act to change the qubit state. First, too strong driving will induce transitions, which in the large 


\section{Quantum efficiency of charge and flux qubit readout}

amplitude limit will be of Landau-Zener type. Secondly, seen from the qubit perspective, the measurement circuit consists of a dissipative transmission line filtered through the oscillator. This will add to the relaxation rate of the qubit, also when not measuring.

\subsection{Non-Adiabatic Corrections}

Equation (11) was derived by diagonalizing the qubit Hamiltonian, a transformation which depends on the state of both oscillator and transmission line. In the adiabatic regime however, it is possible to find eigenvalues and eigenstates of the qubit for each value of the charge on the oscillator and transmission line. Because of the above, the transformation will however also affect the oscillator part of the Hamiltonian, and we must consider this effect and make sure that it is negligible. We begin with the unrotated Hamiltonian for qubit and oscillator (see Eq. (10) )

$$
H=-\frac{E_{e l}(\tilde{n})}{2} \sigma_{z}-\frac{E_{J}}{2} \sigma_{x}+\frac{p^{2}}{2 C}+\frac{\Phi^{2}}{2 L},
$$

where the coupling is in $E_{e l}$, as assumed before. Making the unitary transformation to qubit eigenbasis ${ }^{22}$

$$
\tilde{H}=u^{\dagger} H u, \quad u=\left(\begin{array}{cc}
\cos \eta / 2 & -\sin \eta / 2 \\
\sin \eta / 2 & \cos \eta / 2
\end{array}\right), \quad \eta=\operatorname{arccot}\left(\frac{E_{e l}(\tilde{n})}{E_{J}}\right)
$$

diagonalizes the qubit part of $H$, but also affects the oscillator term that contains the phase. At the degeneracy point $n_{0}=1$ the transformation is given to first order in $\kappa$

$$
u \simeq \frac{1}{\sqrt{2}}\left[\left(\begin{array}{cc}
1 & 1 \\
-1 & 1
\end{array}\right)+\frac{x}{2}\left(\begin{array}{cc}
1 & -1 \\
1 & 1
\end{array}\right)\right]
$$

where $x=4 E_{c} \kappa \tilde{n} / E_{J}$. The term that we have disregarded when deriving Eq. (11) is thus

$$
u^{\dagger} \frac{\Phi^{2}}{2 L} u=\frac{1}{2}\left(\begin{array}{cc}
0 & 1 \\
-1 & 0
\end{array}\right)\left[x, \frac{\Phi^{2}}{2 L}\right]=\frac{i \hbar}{2} \dot{x}\left(\begin{array}{cc}
0 & 1 \\
-1 & 0
\end{array}\right)
$$

which would induce transitions in the qubit. It must therefore be compared with the natural transition energy in the qubit, given by the level splitting 


\section{Tornberg and G. Johansson}

$E_{J}$. Since we explicitly assume both small amplitude $4 \kappa \delta n E_{c} \ll E_{J}$ and low frequency $\hbar \omega \ll E_{J}$ of the driving, the ratio is given by

$$
\frac{4 \kappa E_{c} \hbar \omega \delta n}{E_{J}} \frac{1}{E_{J}} \ll 1
$$

which is indeed negligible within our approximation. We note the similarity of the above condition and the one needed for neglecting Landau-Zener(LZ) transitions. ${ }^{23}$ The LZ transition rate is given by

$$
\Gamma_{L Z} \propto e^{-2 \pi E_{J}^{2} / \hbar v}, \quad v=\partial_{t} E_{e l},
$$

which thus also can be neglected if the condition in Eq. (20) is fulfilled. For the flux qubit the corresponding condition for small amplitude driving is

$$
\frac{M|\langle\phi\rangle| \Phi}{\Delta L_{J} L} \ll 1
$$

In Sec. 8. we will find that the quantum efficiency is independent of the drive strength. But the speed of the measurement is proportional to the power of the drive, as we will see in Sec 6. Thus, in a real experiment one would like to drive as strongly as possible, but one should then make sure not to violate Eqs. (201) or (22).

\subsection{Qubit Relaxation}

Although the pure dephasing induced by the transversely coupled oscillator circuit vanishes to first order in the oscillator-qubit coupling, the qubit relaxation rate $\Gamma_{1}$ has a first order contribution. Thus we evaluate the relaxation rate using standard weak coupling expressions, ${ }^{22}$ giving that it is proportional to the real part of the impedance seen from the qubit, at the qubit frequency $\omega_{q b}$. For the simple oscillator circuits shown in Figs. 1]and [2 with a low $Q$, the relaxation induced by the transmission line can easily dominate over other relaxation mechanisms. The solution to this apparent problem is to add a non-dissipative low-pass filter between the transmission line and the oscillator. Due to the large frequency difference between the oscillator and qubit, this is straightforward. The high-frequency electromagnetic environment responsible for qubit relaxation can be designed independently of the low frequency environment which should let the readout signal through. In principle, a commercially available pi-filter can improve the relaxation rate by a factor of 1000 , while not affecting the low frequency measurement properties. For realistic designs ${ }^{14}$ this is well beyond the point where other sources of relaxation will dominate. 


\section{Quantum efficiency of charge and flux qubit readout}

\section{SYSTEM DYNAMICS}

The questions that we address in this paper concern the efficiency of read-out by probing the quantum capacitance (inductance) of a charge (flux) qubit. The basic idea is that we send a pulse through the transmission line, which will be reflected by the combined oscillator-qubit circuit. In our approximation the pulse will see two different harmonic oscillators, depending on the qubit state. While the information about the state of the qubit state thus is transferred to the reflected pulse, the unavoidable photon number fluctuations in the pulse will dephase the qubit. The statistics of these fluctuations is determined by the way the pulse is generated, and the dynamics of the transmission line coupled to a harmonic oscillator. We thus start out by calculating the dynamics of the free transmission line. Given this, the qubit-oscillator circuit will act as a boundary condition to this solution.

\subsection{Equations of Motion: Transmission Line}

The time-evolution for the transmission line degrees of freedom is governed by the $H_{T L}$ in Eq. (9). For $x>0$ we consider the transmission line phases and charges in the limit $\Delta x \rightarrow 0$. In this limit the Heisenberg equations yield the coupled equations, two for each $i>0$

$$
\begin{aligned}
\dot{p}_{i} & =\left[p_{i}, \frac{\Delta x}{2 L_{T}} \frac{\left(\Phi_{i+1}-\Phi_{i}\right)^{2}-\left(\Phi_{i}-\Phi_{i-1}\right)^{2}}{(\Delta x)^{2}}\right] \rightarrow \frac{\mathrm{d} x}{L_{T}} \frac{\partial^{2} \Phi_{i}}{\partial x^{2}}, \\
p_{i} & =C_{T} \mathrm{~d} x \dot{\Phi}_{i} .
\end{aligned}
$$

Combining these two equations to one we see that the phase in the transmission lines obeys the massless scalar Klein-Gordon equation

$$
\frac{\partial^{2} \Phi}{\partial t^{2}}-\frac{1}{L_{T} C_{T}} \frac{\partial^{2} \Phi}{\partial x^{2}}=0
$$

which indicate the bosonic nature of the transmission line excitations. Equation (24) has a solution in the form of right and leftgoing travelling waves which formally can be written as

$$
\Phi=\Phi^{i n}\left(\frac{x}{v}+t\right)+\Phi^{\text {out }}\left(-\frac{x}{v}+t\right)
$$

where $v=1 / \sqrt{L_{T} C_{T}}$ is the velocity of the waves in the transmission line. By differentiating Eq. (25) we can get a relation between the partial derivatives $^{17}$

$$
-\frac{1}{L_{T}} \frac{\partial \Phi}{\partial x}=\sqrt{\frac{C_{T}}{L_{T}}}\left(\frac{\partial \Phi}{\partial t}-2 \frac{\partial \Phi^{i n}}{\partial t}\right) .
$$




\section{Tornberg and G. Johansson}

The spatial derivative of the probing field at $x=0$ together with Eq. (26) will later provide us with the coupling between circuit and transmission line. It is however natural to first derive the statistics of the transmission line and then go on to the circuit dynamics.

\subsection{Expressing the Transmission Line Using Creation and Annihilation Operators}

The Klein-Gordon equation of motion is easily solved by introducing the Fourier transformed operators in the transmission line

$$
\Phi(x, t)=\frac{1}{\sqrt{2 \pi}} \int_{-\infty}^{\infty} \Phi(k, t) e^{i k x} d k
$$

and we get an ordinary differential equation for each value of the wavenumber $k$. Solving Eq. (24) is reduced to the problem of solving

$$
\ddot{\Phi}(k, t)+v^{2}|k|^{2} \Phi(k, t)=0,
$$

which is the classical equation of motion for a harmonic oscillator with the dispersion relation $\omega_{k}=v|k|$. In analogy with the ordinary harmonic oscillator it is convenient to work with the creation and annihilation operators $a$ and $a^{\dagger}$ instead of charges and phases. However, since we are dealing with a field equation each Fourier component is treated independently and we write $^{24}$

$$
\Phi(k, t)=\sqrt{\frac{\hbar}{2 C_{T} \omega_{k}}}\left(a_{k}(t)+a_{-k}^{\dagger}(t)\right)
$$

where $a$ and $a^{\dagger}$ obey the canonical commutation relations $\left[a_{k}, a_{k^{\prime}}^{\dagger}\right]=\delta\left(k-k^{\prime}\right)$ and $\left[a_{k}, a_{k^{\prime}}\right]=0$, which give the statistics of the transmission line excitations (photons). The harmonic oscillator nature of the transmission line makes the phase of the creation and annihilation operators rotate with angular frequency $\omega_{k}, a_{k}(t)=a_{k} e^{-i \omega_{k} t}, a_{k}^{\dagger}(t)=a_{k}^{\dagger} e^{i \omega_{k} t}$ and we arrive at the final expression for $\Phi$ in terms of $a_{k}$ and $a_{k}^{\dagger}$

$$
\Phi(x, t)=\sqrt{\frac{\hbar}{4 \pi C_{T}}} \int_{-\infty}^{\infty} \frac{d k}{\sqrt{\omega_{k}}}\left(a_{k} e^{-i\left(\omega_{k} t-k x\right)}+a_{k}^{\dagger} e^{i\left(\omega_{k} t-k x\right)}\right) .
$$

In our calculations, we use the in-(out) formalism introduced in Eq. (25), to derive the circuit scattering matrix. For this purpose it is convenient to split the integral in Eq. (30) into positive and negative wavevectors, which 


\section{Quantum efficiency of charge and flux qubit readout}

at $x=0$ can be recognized as the in and out-fields of Eq. (25). As a final remark, one may also write the fields in terms of integrals over frequency giving the normalization

$$
\Phi(x=0, t)=\sqrt{\frac{\hbar Z_{0}}{4 \pi}} \int_{-\infty}^{\infty} \frac{d \omega}{\sqrt{\omega}}\left[a_{\omega} e^{-i \omega t}+\left(a_{\omega}\right)^{\dagger} e^{i \omega t}\right]
$$

where the annihilation and creation operators in frequency space obey the canonical commutation relations $\left[a_{\omega}, a_{\omega^{\prime}}^{\dagger}\right]=\delta\left(\omega-\omega^{\prime}\right)$ and $\left[a_{\omega}, a_{\omega^{\prime}}\right]=0$.

\subsection{Equations of Motion: Charge Qubit}

Considering the adiabatic Hamiltoninan in Eq. (12) and the relation in Eq. (26), the Heisenberg equations of motion for the circuit are

$$
\begin{aligned}
\dot{p_{C}} & =-\frac{1}{L} \Phi_{C}, \quad \dot{p}_{p}=-\sqrt{\frac{C_{T}}{L_{T}}}\left(\frac{\partial \Phi_{p}}{\partial t}-2 \frac{\partial \Phi_{p}^{i n}}{\partial t}\right) \\
\dot{\Phi}_{C} & =\left(D_{23}+g \sigma_{z}\right)\left(p_{C}+p_{p}\right), \quad \dot{\Phi}_{p}=\left(D_{33}+g \sigma_{z}\right) p_{p}+\left(D_{23}+g \sigma_{z}\right) p .
\end{aligned}
$$

This set of four coupled equations can easily be reduced to two, only containing the phase operators of the oscillator and the transmission line. Since the effective circuit only contains linear elements it is straightforward to solve Eq. (32) in Fourier space. The linearity is a direct consequence of the weak coupling between the oscillator and the qubit, where we have replaced the non-linear Josephson element with a linear capacitance. Both the outgoing field and the charge operator coupling to the qubit are expressed in terms of the incoming field

$$
\begin{aligned}
N^{g / e}(\omega) & =\left(D_{23}^{g / e}\right)^{2}+\left(D_{33}^{g / e}-i Z_{0} \omega\right)\left(L \omega^{2}-D_{23}^{g / e}\right) \\
p_{C}^{g / e}(\omega)+p_{p}^{g / e}(\omega) & =\frac{-2 i L \omega^{3}}{N^{g / e}(\omega)} \Phi_{i n}(\omega)=\chi_{g / e}(\omega) \Phi_{i n}(\omega) \\
\Phi_{\text {out }}^{g / e}(\omega) & =\frac{N^{g / e}(-\omega)}{N^{g / e}(\omega)} \Phi_{\text {in }}(\omega)=e^{i \varphi^{g / e}(\omega)} \Phi_{\text {in }}(\omega),
\end{aligned}
$$

where $Z_{0}=\sqrt{L_{T} / C_{T}}$ is the characteristic impedance of the transmission line. We also introduce the short-hand notation $D_{i}^{g / e} \equiv D_{i}+g \sigma_{z}$. Since there is no dissipation in the lumped circuit we have $\left|\Phi^{\text {out }}(\omega)\right|=\left|\Phi^{\text {in }}(\omega)\right|$ and the in- and outgoing field only differ by a state-dependent phase. In the 


\section{Tornberg and G. Johansson}

language of Subs. 5.2. we write Eqs. (33) in the time domain

$$
\begin{aligned}
\phi_{\text {out }}^{g / e}(x, t) & =\sqrt{\frac{\hbar Z_{0}}{4 \pi}} \int_{0}^{\infty} \frac{d \omega}{\sqrt{\omega}}\left(e^{i \phi^{g / e}(\omega)} a_{i n}(\omega) e^{-i \omega(t-x / v)}+\text { h.c. }\right), \\
p^{g / e}(t) & =\sqrt{\frac{\hbar Z_{0}}{4 \pi}} \int_{0}^{\infty} \frac{d \omega}{\sqrt{\omega}}\left(\chi_{g / e}(\omega) a_{i n}(\omega) e^{-i \omega(t-x / v)}+\text { h.c. }\right),
\end{aligned}
$$

where the operators contributing to the charge on the oscillator are written as one operator $p^{g / e}(t) \equiv p_{C}^{g / e}(t)+p_{p}^{g / e}(t)$. We now have the exact dynamics for the combined circuit + transmission line system. However, to get simple analytical expressions for the measurement and dephasing time we need to write the relations $N(\omega)$ and $\chi(\omega)$ on a more simple form. We thus make a Breit-Wigner approximation, see Appendix B. Thus we write $\chi(\omega)$ as

$$
\begin{aligned}
\chi^{g / e}(\omega) & =\frac{\chi_{0}^{g / e}}{1+i 2 Q^{g / e}\left(\frac{\omega-\omega_{0}^{g / e}}{\omega_{0}^{g / e}}\right)} \quad \chi_{0}^{g / e}=\frac{-2}{C_{c} Z_{0} D_{23}^{g / e}}, \\
\omega_{0}^{g / e} & =\sqrt{\frac{D_{23}^{g / e}}{L\left(1+C_{c} D_{23}^{g / e}\right)}} \quad Q^{g / e}=\frac{1}{Z_{0} C_{c}^{2} L\left(\omega_{0}^{g / e}\right)^{3}},
\end{aligned}
$$

where the peak value $\chi_{0}^{g / e}$ is evaluated at the resonance frequency $\omega=\omega_{0}^{g / e}$, and $Q^{g / e}$ is the quality factor of the oscillator.

\subsection{Equations of Motion: Flux Qubit}

The flux-qubit is treated in exact analogy with the charge qubit. The only major difference is that the qubit degree of freedom now couples to the phase, and not charge operator of the oscillator. Given the Hamiltonian in Eq. (14) we solve for $\Phi$ and $\Phi_{p}^{\text {out }}$ in terms of the in-field $\Phi_{p}^{i n}$

$$
\begin{aligned}
N^{g / e}(\omega) & =C\left(1-i C_{c} Z_{0} \omega\right)\left(1-L^{g / e} C \omega^{2}\right)-C_{c} \\
\Phi(\omega) & =\frac{-2 C_{c} C L^{g / e} \omega^{2}}{N^{g / e}(\omega)} \Phi_{p}^{i n}(\omega) \equiv \chi(\omega) \Phi_{p}^{i n}(\omega) \\
\Phi_{p}^{\text {out }}(\omega) & =\frac{N^{g / e}(-\omega)}{N^{g / e}(\omega)} \Phi_{p}^{i n}(\omega)=e^{i \varphi^{g / e}(\omega)} \Phi_{i n}(\omega),
\end{aligned}
$$

where $\chi(\omega)$ for the flux qubit can be written on the exact same Breit-Wigner form as in Eq. (35). The only difference lies in the parameters describing the peak height, width and resonance frequency. In this case they are

$$
\chi_{0}^{g / e}=-i \frac{2 C_{c} Q^{g / e}}{C}, \quad \omega_{0}^{g / e}=\frac{1}{\sqrt{L^{g / e} C_{\mathrm{eff}}}}, \quad Q^{g / e}=\frac{C^{2}}{Z_{0} C_{c}^{2}} \sqrt{\frac{L^{g / e}}{C_{\mathrm{eff}}}},
$$




\section{Quantum efficiency of charge and flux qubit readout}

where $C_{\text {eff }}=C /\left(1-C_{c} / C\right)$.

\subsection{Phase of the Reflected Signal}

We now have a full quantum description of oscillator and qubit dynamics in terms of the incoming field. The interaction can be written on a simple form and we can use this to derive simple analytical expressions for the quantum efficiency for the different qubits. Using the Breit-Wigner approximation, the reflected phase has the following simple form

$$
\varphi_{r}^{g / e}=-2 \arctan \left(\frac{2 Q^{g / e}\left(\omega-\omega_{0}^{g / e}\right)}{\omega_{0}^{g / e}}\right) .
$$

The Breit-Wigner approximation rely on the presence of a relative pronounced resonance in the function $\chi(\omega)$, implying an oscillator with a finite quality factor. We emphasize however that this need not to be the case. Numerical calculations, using the exact form for $N(\omega)$ and $\chi(\omega)$ also give a quantum efficiency $\eta \simeq 1$. In Sec. 9. we calculate $\eta$ without the presence of the oscillator, thereby taking away the resonance altogether. Even in this case, we find a quantum efficiency of unity.

\section{DETECTING THE QUBIT STATE BY HOMODYNE DETECTION}

The interaction between qubit and oscillator allows for an indirect measurement of the qubit state. Since the interaction is purely reactive and no dissipation is present in the process, all information about the qubit is contained in the phase of the reflected signal. To analyze the quantum efficiency of such a read-out we use standard techniques from quantum optics and describe it as a homodyne measurement. ${ }^{15}$ In this setup, the reflected signal from the circuit is mixed with a phase-locked local oscillator field using a linear mixer, a beam-splitter combined with a photon detector in the optical case. By detecting the power of this mixed signal, information about the phase can be extracted. Labelling the mixed signal with the operator $b$ gives the field intensity at the detector

$$
N(T)=\int_{0}^{T} d t b^{\dagger}(t) b(t)
$$

Detecting intensity is equivalent to detecting photons, a destructive process where photons are converted into current by a photoamplifier. Our signal 


\section{Tornberg and G. Johansson}

will thus be given by the annihilation operator at the detector

$$
b(t)=r \alpha(t)+r v(t)+t a(t),
$$

which contains the average $\alpha$ and residual $v$ of the LO mode. The reflected signal from the circuit is given by the operator $a$. The reflection and transmission coefficients $r$ and $t$ are assumed to be real. For optimal efficiency we need that $r \ll 1$ which allows almost all the signal from the circuit to be transmitted to the detector. By amplifying the local oscillator field $r \alpha(t)$ can still be made dominant so that second order contributions in $v(t)$ and $a(t)$ can be neglected in Eq. (39). Both the LO-field and signal are modelled as a Glauber state ${ }^{25}$

$$
|\{\alpha(\omega)\}\rangle=\exp \left(\int d \omega\left[\alpha(\omega)\left(a_{\omega}^{i n}\right)^{\dagger}-\alpha^{*}(\omega) a_{\omega}^{i n}\right]\right)|0\rangle,
$$

where $\alpha(\omega)$ is the Fourier-transform of our drive signal, and $|0\rangle$ is the continuum vacuum field $a_{\omega}|0\rangle=0$. The drive source is nearly monochromatic and has a narrow bandwidth distribution $\left(\Gamma_{d} \ll \omega_{d}\right)$ which is Gaussian in frequency

$$
\alpha(\omega)=\alpha_{0} \frac{\omega_{d}}{\Gamma_{d}} \frac{e^{-\left(\omega-\omega_{d}\right)^{2} / 2 \Gamma_{d}^{2}}}{\sqrt{\omega}},
$$

where $\alpha_{0}$ is a dimensionless constant and $\omega_{d}$ is the drive frequency. At the detector we observe the quantum mechanical expectation value of the operator $N$. Assuming that the deviation $v$ from the average LO signal is in a vacuum state, this is given by

$$
\langle N(T)\rangle=T\left(r^{2} \alpha_{L O}^{2}+2 \operatorname{tr} \alpha_{L O} \cos (\phi+\theta)\right),
$$

where $\phi$ and $\theta$ are the phases of the signal and LO-field respectively. The first term only contains information about the LO-field and does not contribute to the phase information. We thus take the second term as the signal at the detector $S$. The standard deviation of the photon number operator is

$$
\Delta N=r \alpha_{L O} \sqrt{T}
$$

which we take to be the signal noise $N o$. The measurement time $t_{m s}$ is defined as the time for which the signal to noise ratio is one. Choosing $\theta+\left(\phi_{r}^{g}+\phi_{r}^{e}\right) / 2=\pi / 2$, and $t \simeq 1$ it is given by

$$
t_{m s}=\frac{1}{\Gamma_{i n}} \frac{1}{4 \sin ^{2}\left[\frac{\varphi_{r}^{g}-\varphi_{r}^{e}}{2}\right]},
$$

where $\Gamma_{i n}$ is rate of photons sent down to the circuit by the drive. We now have a formula for the measurement time, determined by the driving strength and the difference in the reflected phase between the two qubit states. This we may compare with the measurement induced dephasing. 


\section{Quantum efficiency of charge and flux qubit readout \\ 7. QUBIT DEPHASING}

The dephasing rate $\Gamma_{\varphi}$ is defined as the rate with which the qubit looses its phase coherence. That is how fast the off-diagonal matrix elements in the reduced qubit density matrix go to zero. Since the phase coherence is the heart of all quantum computation, the qubit is useless after a time given by the dephasing time $1 / \Gamma_{\varphi}$. The short dephasing time is the major bottleneck preventing the progress in solid state quantum computing. The reason for such short life-times are the ever present strong couplings between qubit and environment inducing all kinds of fluctuations in the qubit. We must therefore see to that all additional sources of decoherence are minimized to reduce this effect. One such source is the transmission line that we have attached to the qubit for read-out. Photons are the elementary excitations of energy in the transmission line and fluctuations in these will dephase the qubit. In this section we calculate the dephasing rate for two different cases. In the first case the state of the in-field is set to a Glauber state as was done in Sec. 6. to simulate the field generated by a monochromatic source used for readout. In the second case a thermal distribution of photons is used to simulate the dephasing due to the small but finite temperature in the experimental setup, which occurs also when not reading out.

\subsection{Dephasing from Field Correlators}

We begin by considering a general pure state characterizing the qubit and oscillator

$$
|\Psi\rangle=a|g\rangle \otimes|\psi\rangle_{g}^{\mathrm{osc}}+b|e\rangle \otimes|\psi\rangle_{e}^{\mathrm{osc}},
$$

where $a$ and $b$ are complex numbers fulfilling $|a|^{2}+|b|^{2}=1$. At the degeneracy point the Hamiltonian describing the qubit and interaction with the oscillator is given by

$$
H=\frac{E_{J}}{2} \sigma_{z}+\frac{g}{2} \sigma_{z} P^{2}=\underbrace{\frac{E_{J}}{2} \sigma_{z}+\frac{g}{2} \sigma_{z}\left\langle P^{2}\right\rangle}_{H_{0}}+\frac{g}{2} \sigma_{z} p^{2},
$$

where we incorporate the average value of $p$ into the Hamiltonian $H_{0}$. Our first task is to calculate the time evolution of the $\sigma_{+}$operator. We use the charge qubit as example, but both the formalism and final result apply to the flux qubit as well. For our purposes it is convenient to make a unitary transformation to the rotating frame of the unperturbed qubit system $H_{0}$

$$
O_{I}(t)=e^{-\frac{i}{\hbar} \int H_{0} d t} O_{H}(t) e^{\frac{i}{\hbar} \int H_{0} d t},
$$




\section{Tornberg and G. Johansson}

where $H_{0}$ is the unperturbed Hamiltonian. In this frame the equation of motion for the $\sigma_{+}$-operator is

$$
\begin{aligned}
\frac{\partial \sigma_{+}}{\partial t}= & -i \frac{g}{\hbar} p^{2}(t) \sigma_{+}(0)-\frac{g^{2}}{\hbar^{2}} \int_{0}^{t}\left[\sigma_{+}(t) p^{2}\left(t^{\prime}\right) p^{2}(t)+p^{2}(t) \sigma_{+}(t) p^{2}\left(t^{\prime}\right)+\right. \\
& \left.+p^{2}\left(t^{\prime}\right) \sigma_{+}(t) p^{2}(t)+p^{2}(t) p^{2}\left(t^{\prime}\right) \sigma_{+}(t)\right] d t^{\prime}
\end{aligned}
$$

to second order in $g$. Moreover, the evolution of $\sigma_{+}$is assumed to be slow on the time-scale of the oscillator. The dephasing is now given by multiplying Eq. (49) by the state in Eq. (46) from the left and right and tracing out the oscillator degrees of freedom, giving an equation of motion for $a b^{*}$, the off-diagonal element in the reduced density matrix

$$
\begin{aligned}
\frac{\partial\left(a b^{*}\right)}{\partial t}= & -a b^{*} \frac{g^{2}}{4 \hbar^{2}} \int_{0}^{t}\left[\left\langle p_{g}^{2}\left(t^{\prime}\right) p_{g}^{2}(t)\right\rangle+\left\langle p_{e}^{2}(t) p_{g}^{2}\left(t^{\prime}\right)\right\rangle+\right. \\
& \left.+\left\langle p_{e}^{2}\left(t^{\prime}\right) p_{g}^{2}(t\rangle\right)+\left\langle p_{e}^{2}(t) p_{e}^{2}\left(t^{\prime}\right)\right\rangle\right] d t^{\prime} .
\end{aligned}
$$

The index on the $p^{2}$-operator indicates the state of the field. We now have an expression for the dephasing rate. The only remaining task is to put the in-field in a given state and calculate the two-time correlators of Eq. (50).

\subsection{Dephasing Induced by the Read-Out}

In the read-out process the oscillator state in Eq. (46) is a coherent Glauber state, see Eq. 411)

$$
|\Psi\rangle=a|g\rangle \otimes|\alpha(\omega)\rangle_{g}+b|e\rangle \otimes|\alpha(\omega)\rangle_{e} .
$$

The oscillator state is now an eigenstate of the annihilation operator which makes the calculation of the correlators in Eq. (50) a matter of putting the creation and annihilation operators in normal order. Using the narrow bandwidth of the drive as given in Eq. (42) the correlators are found to be

$$
\begin{array}{r}
\left\langle p_{\alpha}^{2}\left(t^{\prime}\right) p_{\beta}^{2}(t)\right\rangle=4\left\langle p_{\alpha}(t)\right\rangle\left\langle p_{\beta}\left(t^{\prime}\right)\right\rangle \frac{\hbar Z_{0}}{4 \pi} \int_{0}^{\infty} \frac{\chi_{\alpha}(\omega) \chi_{\beta}^{*}(\omega)}{\omega} e^{-i \omega\left(t-t^{\prime}\right)}+ \\
+2 \frac{\hbar^{2} Z_{0}^{2}}{16 \pi^{2}} \int_{0}^{\infty} \int_{0}^{\infty} d \omega_{1} d \omega_{2} \frac{\chi_{\alpha}\left(\omega_{1}\right) \chi_{\beta}^{*}\left(\omega_{1}\right)}{\omega_{1}} \frac{\chi_{\alpha}\left(\omega_{2}\right) \chi_{\beta}^{*}\left(\omega_{2}\right)}{\omega_{2}} e^{-i\left(\omega_{1}+\omega_{2}\right)\left(t-t^{\prime}\right)} .
\end{array}
$$

The dephasing rate is given by taking the zero frequency Fourier transform. We make the variable transformation $t-t^{\prime}=\tau$ and extend the upper limit of the integral to infinity, an approximation which is valid if we limit ourselves to times longer than the field correlation time. Neglecting the imaginary 


\section{Quantum efficiency of charge and flux qubit readout}

part of the transform, which only gives a small renormalization to the qubit energy splitting, we get the simple expression

$$
\operatorname{Re}\left\{\int_{0}^{\infty}\left\langle p_{\alpha}^{2}\left(t^{\prime}\right) p_{\beta}^{2}(t)\right\rangle d t^{\prime}\right\}=\Gamma_{i n} \frac{\hbar^{2} Z_{0}^{2}}{2 \omega_{d}} \frac{\left|\chi_{\alpha}\left(\omega_{d}\right)\right|^{2}\left|\chi_{\beta}\left(\omega_{d}\right)\right|^{2}}{\omega_{d}} .
$$

where $\Gamma_{i n}$ is the rate of photons sent down the transmission line and $\omega_{d}$ is the drive frequency. Solving Eq. (50) together with the result from above. the off-diagonal element will exhibit an exponential decay with the dephasing rate given by

$$
\Gamma_{\varphi}=\Gamma_{i n} \frac{g^{2} Z_{0}^{2}}{8 \omega_{d}^{2}}\left(\left|\chi_{g}\left(\omega_{d}\right)\right|^{2}+\left|\chi_{e}\left(\omega_{d}\right)\right|^{2}\right)^{2}
$$

This result is valid for both charge and flux qubits, choosing the correct expressions for coupling $g=g_{C}=C_{Q} / C_{o s c}^{2}$ and $g=g_{L}=L_{Q} / L^{2}$, as well as for the transfer function $\chi_{g / e}(\omega)$ from Eqs. (33) and (36). As expected the dephasing rate increases with the drive power. We also note that the rate is inversely proportional to the drive frequency squared, not surprising since the dephasing is given by the zero-frequency fluctuations in the field.

\section{3. $\quad$ Finite Temperature Dephasing}

Because of the non-zero temperature in the environment, photons in the transmission line will be thermally excited and contribute to the dephasing of the qubit. It is desireble to reduce this effect by an efficient design. We are therefore interested to see how this dephasing scales with the different circuit parameters. The state of the oscillator in Eq. (46) is given by a thermal distribution of photons. The correlators in Eq. (50) are characterized by

$$
\left\langle a_{\omega}^{\dagger} a_{\omega^{\prime}}\right\rangle=\delta\left(\omega-\omega^{\prime}\right) n(\omega), \quad n(\omega)=\frac{1}{e^{\beta \hbar \omega}-1},
$$

where $n(\omega)$ is the Bose-occupation number. Given this it is straightforward to calculate the dephasing rate as was done in Subs. 7.2. Following Eq. (50) we calculate the correlator

$$
\begin{aligned}
& \left\langle p_{\alpha}^{2}(t) p_{\beta}^{2}\left(t^{\prime}\right)\right\rangle=\left\langle p_{\alpha}^{2}\right\rangle\left\langle p_{\beta}^{2}\right\rangle+2 \frac{\hbar^{2} Z_{0}^{2}}{16 \pi^{2}} \int_{0}^{\infty} \int_{0}^{\infty} d \omega d \omega^{\prime} \times \\
\times & {\left[\frac{\chi_{\alpha}(\omega) \chi_{\beta}^{*}(\omega)}{\omega}(n(\omega)+1) e^{-i \omega\left(t-t^{\prime}\right)}+\frac{\chi_{\alpha}^{*}(\omega) \chi_{\beta}(\omega)}{\omega} n(\omega) e^{i \omega\left(t-t^{\prime}\right)}\right] \times } \\
\times & {\left[\frac{\chi_{\alpha}\left(\omega^{\prime}\right) \chi_{\beta}^{*}\left(\omega^{\prime}\right)}{\omega^{\prime}}\left(n\left(\omega^{\prime}\right)+1\right) e^{-i \omega^{\prime}\left(t-t^{\prime}\right)}+\frac{\chi_{\alpha}^{*}\left(\omega^{\prime}\right) \chi_{\beta}\left(\omega^{\prime}\right)}{\omega^{\prime}} n\left(\omega^{\prime}\right) e^{i \omega^{\prime}\left(t-t^{\prime}\right)}\right], }
\end{aligned}
$$




\section{Tornberg and G. Johansson}

where the first term vanishes since the $p^{2}$-operator has zero average. The dephasing rate is given by the Fourier transform of Eq. (56). As in Subs. 7.2. we make the substitution $\tau=t-t^{\prime}$ and ignore the imaginary part of the integral, giving

$$
\Gamma_{\varphi}=\frac{Z_{0}^{2} g^{2}}{16 \pi} \int_{0}^{\infty}\left(\left|\chi_{g}(\omega)\right|^{2}+\left|\chi_{e}(\omega)\right|^{2}\right)^{2}(n(\omega)+1) n(\omega) \frac{d \omega}{\omega^{2}},
$$

valid for both charge and flux qubits. Analytically we may proceed in the Breit-Wigner approximation of the transfer functions $\chi_{g / e}(\omega)$. We want to compare this dephasing rate with other dephasing mechanism affecting the qubit. For this purpose it is sufficient to make the approximation $\left|\chi_{g}\right| \approx$ $\left|\chi_{e}\right|$, and ignore effects of the quantum capacitance. Given that the rest of the integrand varies slowly compared to $\chi(\omega)$ we arrive at the following expression for the dephasing rate for the charge qubit

$$
\Gamma_{\varphi}=\omega_{0} Q \frac{C_{Q}^{2}}{C_{o s c}^{2}}\left(n\left(\omega_{0}\right)+1\right) n\left(\omega_{0}\right),
$$

where $\omega_{0}=1 / \sqrt{L C_{\text {osc }}}$ and $Q=1 /\left(Z_{0} C_{c}^{2} L \omega_{0}\right)$ is the approximate resonance frequency and $\mathrm{Q}$ value. A similar expression was found by Bertet et. al. considering a flux qubit coupled to a SQUID, in the high Q regime. ${ }^{26}$ As expected the dephasing rate scales badly with the temperature due to the bosonic nature of the transmission line excitations. However we see that a low oscillator $Q$-value protects the qubit from dephasing, basically by pushing the noise spectrum up to higher frequencies.

\section{QUANTUM EFFICIENCY}

There is always a trade-off between speed in read-out and how fast the qubit is dephased. It is impossible to obtain information about the state of the qubit faster than the phase coherence between the states are lost. Given a longitudinal coupling between qubit and oscillator and a probing field in a coherent Glauber state, which is narrow in frequency distribution, we have derived the following general expressions for the dephasing and measurement rate

$$
\begin{aligned}
\Gamma_{\varphi} & =\Gamma_{i n} \frac{\left(g Z_{0}\right)^{2}}{8 \omega_{d}^{2}}\left(\left|\chi_{g}(\omega)\right|^{2}+\left|\chi_{e}(\omega)\right|^{2}\right)^{2} \\
t_{m s}^{-1} & =4 \Gamma_{i n} \sin ^{2}\left(\frac{\delta \phi}{2}\right)
\end{aligned}
$$

where $g$ is the coupling between qubit and oscillator degrees of freedom and $\chi(\omega)$ describes the relation between charge (phase) of the oscillator and the 


\section{Quantum efficiency of charge and flux qubit readout}

in field. We now assume that the function $\chi(\omega)$ can be written on BreitWigner form as in Eq. (35). Using this, we get explicit expressions for the dephasing rate in terms of circuit parameters

$$
\Gamma=\Gamma_{i n} \frac{\left(g Z_{0}\right)^{2}}{8 \omega_{d}^{2}}\left(\frac{\left(\chi_{0}^{g}\right)^{2}}{1+4\left[\frac{Q^{g}}{\omega_{0}^{g}}\left(\omega_{0}^{g}-\omega\right)\right]^{2}}+\frac{\left(\chi_{0}^{e}\right)^{2}}{1+4\left[\frac{Q^{e}}{\omega_{0}^{e}}\left(\omega_{0}^{e}-\omega\right)\right]^{2}}\right)^{2}
$$

and the phase difference between the two qubit states gets the simple form

$$
\delta \phi=2 \arctan \left(\frac{2 Q^{g}}{\omega_{0}^{g}}\left(\omega-\omega_{0}^{g}\right)\right)-2 \arctan \left(\frac{2 Q^{e}}{\omega_{0}^{e}}\left(\omega-\omega_{0}^{e}\right)\right) .
$$

Choosing the drive frequency to be centered between the two resonance frequencies $\omega_{d}=\frac{\omega_{0}^{g}+\omega_{0}^{e}}{2}$, these expressions simplify to

$$
\begin{aligned}
\Gamma_{\varphi} & =\Gamma_{i n} \frac{\left(g Z_{0}\right)^{2}}{8 \omega_{d}^{2}}\left[\frac{\left(\chi_{0}^{g}\right)^{2}}{x_{g}\left(x_{g}^{-1}+x_{g}\right)}+\frac{\left(\chi_{0}^{e}\right)^{2}}{x_{e}\left(x_{e}^{-1}+x_{e}\right)}\right]^{2} \\
t_{m s}^{-1} & =4 \Gamma_{i n} \sin ^{2}\left(\arctan x_{e}+\arctan x_{g}\right)
\end{aligned}
$$

where $x_{g / e}=Q^{g / e}\left(\omega_{0}^{e}-\omega_{0}^{g}\right) / \omega_{0}^{g / e}$. Defining the quantum efficiency as $\eta=$ $\Gamma_{m s} /\left(2 \Gamma_{\phi}\right)$ and using Eq. (62) the efficiency is given in terms of general parameters in the Breit-Wigner approximation. Using the parameters given for the charge and phase qubit in Eqs. (35) and (37) respectively and neglecting second order effects in the qubit-oscillator coupling (small parameter is $C_{Q}^{2} / C_{\text {osc }}^{2}$ and $L_{Q}^{2} / L^{2}$ respectively) we get a quantum efficiency $\eta=1$ for both charge and flux qubit.

For another choice of drive frequency, we find an efficiency below unity. For these non-optimal frequencies, the charge magnitude on the oscillator differ for the two different qubit states. In this case, since the time to build up a charge on the oscillator depend on the qubit state, the time response of the oscillator will differ. Thus some information of the qubit state will be encoded in the response time, and since our setup only detects the phase, it misses this information, making the efficiency decrease. For the optimal drive frequency above, the charge on the oscillator is the same regardless of qubit state, and all information is encoded in the signal phase.

In a more realistic situation, the qubit will of course be subject to various additional sources of decoherence, i.e. thermal photons in the read-out lines and charge fluctuations in the environment, and these will reduce the quantum efficiency. Taking these sources into account is however beyond the scope of this paper. 


\section{Tornberg and G. Johansson}

\section{MEASURING THE QUANTUM CAPACITANCE WITHOUT OSCILLATOR}

The calculation of the quantum efficiency for the charge and flux qubit was done assuming a rather sharp resonance in the function $\chi(\omega)$ approximating it with a Breit-Wigner form. We can however show that the result still holds if we remove the oscillator, thereby taking away the resonance completely. For this purpose we consider the circuit in Fig. 3 where the

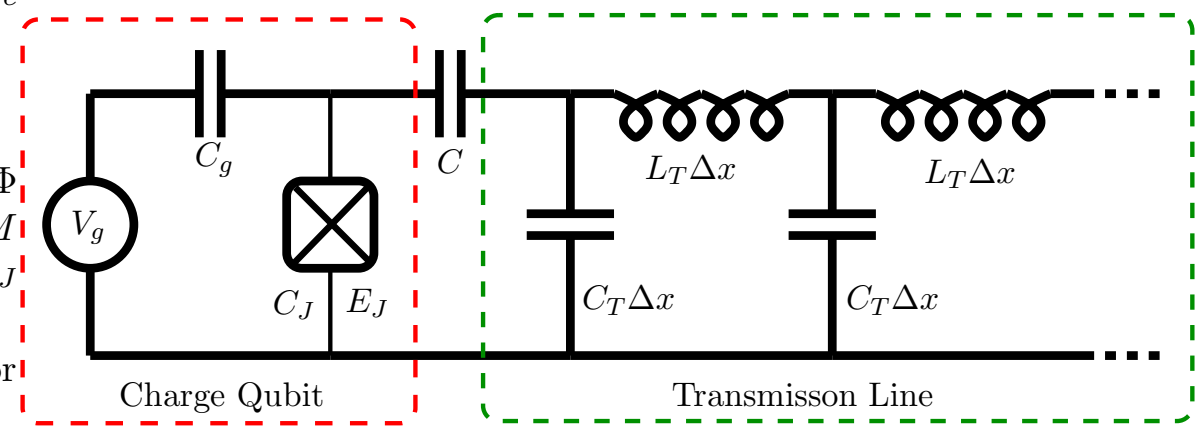

Fig. 3. Cooper-pair box operated as a charge qubit. The read-out circuit only consists of a measurement capacitance $C$, still giving a full quantum efficiency $\eta=1$.

charge qubit is directly coupled to the transmission line via the capacitor $C$. For this circuit the Hamiltonian is given by

$$
H=\frac{p_{1}^{2}}{2 C_{\mathrm{g} / \mathrm{e}}}+\frac{1}{\Delta x} \sum_{i}\left(\frac{\left(p_{(i+1)}^{p}\right)^{2}}{2 C_{T}}+\frac{\left(\Phi_{i+1}^{p}-\Phi_{i}^{p}\right)^{2}}{2 L_{T}}\right),
$$

where $C_{\mathrm{g} / \mathrm{e}}=C /\left(1+g C \sigma_{z}\right)$ and $g=\left(8 E_{C}^{2}\right) /\left(e^{2} E_{J}\right)$ is the coupling between qubit and transmission line. The functions relating the in-field with the charge at the transmission-line boundary and the reflected signal are

$p(\omega)=\frac{2 \omega C_{\mathrm{g} / \mathrm{e}} Z_{C}(\omega)}{i Z_{C}(\omega)+Z_{0}} \Phi^{i n}(\omega) \equiv \chi(\omega) \Phi^{i n}(\omega), \Phi^{\text {out }}(\omega)=\frac{i Z_{C}(\omega)-Z_{0}}{i Z_{C}(\omega)+Z_{0}} \Phi^{i n}(\omega)$,

where $Z_{C}(\omega)=1 /\left(\omega C_{\mathrm{g} / \mathrm{e}}\right)$ is the impedance of the effective qubit-capacitor circuit. As in Sec. 8. we use a narrow bandwidth drive signal with drive frequency $\omega_{d}$ and Eq. (59) to calculate the dephasing and measurement rate

$$
\begin{aligned}
\Gamma_{\varphi} & =\Gamma_{i n} \frac{\left(g Z_{0}\right)^{2}}{8 \omega_{d}^{2}}\left(\frac{4\left(\omega_{d} C_{g}\right)^{2}}{1+\left(\omega_{d} C_{g} Z_{0}\right)^{2}}+\frac{4\left(\omega_{d} C_{e}\right)^{2}}{1+\left(\omega_{d} C_{e} Z_{0}\right)^{2}}\right)^{2}, \\
t_{m s}^{-1} & =4 \Gamma_{i n} \sin ^{2}\left(\arctan \left(\omega_{d} Z_{0} C_{e}\right)-\arctan \left(\omega_{d} Z_{0} C_{g}\right)\right) .
\end{aligned}
$$




\section{Quantum efficiency of charge and flux qubit readout}

In this case a quantum efficiency of unity is also obtained, neglecting second order effects in the qubit-transmission line coupling $g$. This is however of more academic interest considering that typical parameters $Z_{0}=50 \mathrm{Ohm}$, $C_{g}-C_{e}=1 \mathrm{fF}$, give a very small phase-difference on the order of $\delta \varphi \sim 10^{-4}$, even for a high drive frequency of $\omega_{d}=1 \mathrm{GHz}$. A too small phase difference implies a small signal, which in a real setup will drown in the amplifier noise.

\section{CONCLUSION}

We have described dispersive charge and flux qubit readout using a slow oscillator as a quantum measurement process. Our main approximation is that the qubit follows the oscillator adiabatically, and we give conditions for this approximation to hold. Within this approximation the qubit acts as an effective linear circuit element in the oscillator circuit. For the charge qubit it is a capacitance for the flux qubit an inductance. The quantum dynamics of the effectively linear oscillator-transmission line is readily solved, and we get expression for all quantities in terms of the input field of the transmission line. Especially we treat the coupling between the oscillator and transmission line nonperturbatively.

We use this formalism to calculate the quantum efficiency of the readout. This is found to be unity, within our approximation, for both charge and flux qubit readout. As an extra example we also find unit quantum efficiency for a charge qubit directly coupled to a transmission line.

\section{Appendix A. THE LEGENDRE TRANSFORM}

Given the Lagrangian as presented in Eq. (4), and neglecting the potential part,

$$
L=\frac{1}{2} \dot{\phi}_{i} C_{i k} \dot{\phi}_{k}-a_{k} \dot{\phi}_{k}
$$

we define the conjugated momenta according to

$$
p_{j} \equiv \frac{\partial L}{\partial \dot{\phi}_{j}}=\dot{\phi}_{i} C_{i j}-a_{j}
$$

Given this, the Hamiltonian is obtained by a Legendre transformation of the Lagrangian

$$
\begin{aligned}
H & =p_{j} \dot{\phi}_{j}-L=p_{j}\left(p_{k}+a_{k}\right)\left(C^{-1}\right)_{k j}- \\
& -\frac{1}{2}\left(p_{i}+a_{i}\right) \underbrace{\left(C^{-1}\right)_{i j} C_{j k}}_{\delta_{i k}}\left(C^{-1}\right)_{k m}\left(p_{m}+a_{m}\right)+a_{i}\left(p_{k}+a_{k}\right)\left(C^{-1}\right)_{k i}=
\end{aligned}
$$




\section{Tornberg and G. Johansson}

$$
\begin{aligned}
& =p_{j}\left(p_{k}+a_{k}\right)\left(C^{-1}\right)_{k j}-\frac{1}{2}\left(p_{i}+a_{i}\right)\left(C^{-1}\right)_{i m}\left(p_{m}+a_{m}\right)+ \\
& +a_{i}\left(p_{k}+a_{k}\right)\left(C^{-1}\right)_{k i}=\left(p_{i}+a_{i}\right)\left(p_{k}+a_{k}\right)\left(C^{-1}\right)_{k i}- \\
& -\frac{1}{2}\left(p_{i}+a_{i}\right)\left(C^{-1}\right)_{i m}\left(p_{m}+a_{m}\right)=\frac{1}{2}\left(p_{i}+a_{i}\right)\left(C^{-1}\right)_{k m}\left(p_{m}+a_{m}\right),
\end{aligned}
$$

making the derivation of the Hamiltonian a matter of inverting the matrix $\hat{C}$, and subtracting the potential part of the Lagrangian.

\section{Appendix B. BREIT-WIGNER APPROXIMATION; CHARGE QUBIT + OSCILLATOR}

We are interested in the resonant behaviour of the transfer/response functions around $\omega=1 / \sqrt{L C_{o s c}}$. We expand the relevant solution to $N^{g / e}(\omega)=$ 0 to first order in $Z_{0}$

$$
\begin{aligned}
\omega_{\text {res }} & =\sqrt{\frac{D_{23}^{g / e}}{L\left(1+C_{c} D_{23}^{g / e}\right)}}+i Z_{0} \frac{C_{c}\left(D_{23}^{g / e}\right)^{5 / 2}}{\left(1+C_{c} D_{23}^{g / e}\right)^{3 / 2} \sqrt{L}} \equiv \omega_{0}^{g / e}\left(1+i \frac{1}{2 Q^{g / e}}\right), \\
\omega_{0}^{g / e} & =\sqrt{\frac{D_{23}^{g / e}}{L\left(1+C_{c} D_{23}^{g / e}\right)}}, \quad Q^{g / e}=\frac{1}{Z_{0} C_{c}^{2} L\left(\omega_{0}^{g / e}\right)^{3}},
\end{aligned}
$$

leading to

$$
N^{g / e}(\omega) \approx \frac{2 D_{23}^{g / e}}{C_{c} \omega_{0}^{g / e}}\left(\omega-\omega_{0}^{g / e}-i \frac{\omega_{0}^{g / e}}{2 Q^{g / e}}\right)=-i \frac{D_{23}^{g / e}}{Q^{g / e} C_{c}}\left(1+i 2 Q^{g / e} \frac{\omega-\omega_{0}^{g / e}}{\omega_{0}^{g / e}}\right) .
$$

Thus we approximate the transconductance as (assuming $C_{c} Z_{0} \omega_{0} \ll 1$ )

$$
\begin{aligned}
\chi^{g / e}(\omega) & =\frac{-2}{C_{c} Z_{0} D_{23}^{g / e}} \frac{\chi_{0}^{g / e}}{1+i 2 Q^{g / e}\left(\frac{\omega-\omega_{0}^{g / e}}{\omega_{0}^{g / e}}\right)}, \\
\chi_{0}^{g / e} & =\frac{-2}{C_{c} Z_{0} D_{23}^{g / e}} \approx-2 \sqrt{\frac{Q^{g / e}}{L Z_{0} \omega_{0}^{g / e}}},
\end{aligned}
$$

and the reflected phase as

$$
\varphi_{r}^{g / e}=2 \arg \left[\frac{\omega_{0}^{g / e}}{2 Q^{g / e}}-i\left(\omega-\omega_{0}^{g / e}\right)\right]=-2 \arctan \left(\frac{2 Q^{g / e}\left(\omega-\omega_{0}^{g / e}\right)}{\omega_{0}^{g / e}}\right) .
$$




\section{ACKNOWLEDGMENTS}

We thank Chris Wilson, Per Delsing, Tim Duty, Vitaly Shumeiko, Göran Wendin and Margareta Wallquist for valuable discussions. We would also like to thank Matthias Braun for useful comments on the manuscript. This work was supported by the Swedish SSF and VR, and by the EU under the EUROSQIP programme.

\section{REFERENCES}

1. G. Wendin and V.S. Shumeiko, in Handbook of Theoretical and Computational Nanotechnology, eds. M. Rieth and W. Schommers, American Scientific Publishers (2006).

2. G. Johansson, L. Tornberg, V.S. Shumeiko and G. Wendin, J. Phys.: Condens. Matter 18, S901-S920 (2006).

3. Y. Nakamura, Y. A. Pashkin and J. S. Tsai, Nature (London) 398, 786 (1999).

4. O. Astafiev, Yu. A. Pashkin, Y. Nakamura, T. Yamamoto, and J. S. Tsai, Phys. Rev. Lett. 93, 267007 (2004).

5. I. Chiorescu, Y. Nakamura, C.J.P.M. Harmans, J.E. Mooij, Science 299, 1869 (2003).

6. D. Vion, A. Cottet, A. Aassime, P. Joyez, H. Pothier, C. Urbina, D. Esteve and M.H. Devoret, Science 296, 886 (2002).

7. G. Ithier, E. Collin, P. Joyez, P.J. Meeson, D. Vion, D. Esteve, F. Chiarello, A. Shnirman, Y. Makhlin, J. Schriefl, and G. Schön, Phys. Rev. B, 72, 134519 (2005).

8. M. Grajcar, A. Izmalkov, E. Il'ichev, Th. Wagner, N. Oukhanski, U. Hubner, T. May, I. Zhilyaev, H. E. Hoenig, Ya. S. Greenberg, V. I. Shnyrkov, D. Born, W. Krech, H. -G. Meyer, Alec Maassen van den Brink, and M. H. S. Amin, Phys. Rev. B 69, 060501(R) (2004).

9. A. Lupascu, C. J.M. Verwijs, R. N. Schouten, C. J. P.M. Harmans, and J. E. Mooij, Phys. Rev. Lett. 93, 177006 (2004).

10. A. Wallraff, D. I. Schuster, A. Blais, L. Frunzio, J. Majer, M. H. Devoret, S. M. Girvin, and R. J. Schoelkopf, Phys. Rev. Lett. 95, 060501 (2005).

11. T. Duty, G. Johansson, K. Bladh, D. Gunnarsson, C. Wilson, and P. Delsing, Phys. Rev. Lett. 95, 206807 (2005).

12. M. A. Sillanpää, T. Lehtinen, A. Paila, Yu. Makhlin, L. Roschier, and P. J. Hakonen, Phys. Rev. Lett. 95, 206806 (2005).

13. V. Braginski and F. Khalili, Quantum Measurements, Cambridge University Press, (1992).

14. G. Johansson, L. Tornberg and C. Wilson, Phys. Rev. B 74, 100504(R) (2006).

15. C. W. Gardiner, P. Zoller Quantum Noise (Springer-Verlag Berlin Heidelberg, $1991,2000)$.

16. A. Blais, R. Huang, A. Wallraff, S. M. Girvin and R. J. Schoelkopf, Phys. Rev. A 69, 062320 (2004).

17. B. Yurke and J. S. Denker, Phys. Rev. A 29, 1419 (1984). 


\section{Tornberg and G. Johansson}

18. A. Shnirman, G. Schön, and Z. Hermon, Phys. Rev. Lett. 79, 2371 (1997).

19. M. Devoret, Quantum fluctuations in electrical circuits, Les Houches, Session LXIII (1995).

20. H. Goldstein, Classical mechanics, Addison-Wesley, Reading, Massachusetts (1980).

21. J. R. Friedman, V. Patel, W. Chen, S. K. Tolpygo, and J. E. Lukens, Nature, 406, 4345, (2000).

22. Y. Makhlin, G. Schön and A. Shnirman, Rev. Mod. Phys. 73357 (2001).

23. L. Landau, Phys. Z. Sowjetunion 2, 46 (1932); C. Zener, Proceedings of the Royal Society A 137, 696 (1932).

24. M. E. Peskin and D. V. Schröder, An Introduction to Quantum Field theory (Westview Press, 1995).

25. S. M. Barnett and P. M. Radmore Methods in Theoretical Quantum Optics (Oxford University Press, 1997).

26. P. Bertet, I. Chiorescu, G. Burkard, K. Semba, C. J. P. M. Harmans, D. P. DiVincenzo, and J. E. Mooij, Phys. Rev. Lett. 95, 257002 (2005). 